\title{
Mediastinitis por Aspergillus fumigatus en un paciente pediátrico inmunocompetente posterior a una cardiocirugía
}

\author{
Mirta Acuña, Felipe Farfán, Fernanda Cofré y Dona Benadof
}

\section{Aspergillus fumigatus mediastinitis in an immunocompetent pediatric patient after heart surgery}

Postsurgical aspergillosis occurs primarily in immunocompetent patients whose main predisposing factor is the loss of skin and mucosal integrity during surgery. Local infection tends to be destructive and refractory to treatment and relapses are common. It is important to consider aspergillosis in the differential diagnosis of slowly progressive and destructive surgical site infections with negative bacterial cultures. We present the case of a child who developed Aspergillus fumigatus mediastinitis months after heart surgery.

Key words: Aspergillus fumigatus, mediastinitis, aspergillosis.

Palabras clave: Aspergillus fumigatus, mediastinitis, aspergilosis post-quirúrgica.

\section{Introducción}

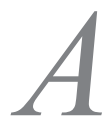
spergillus spp. es un hongo ubicuo y saprofítico que tiene un rol principal en el metabolismo del carbono y nitrógeno en la naturaleza. En forma primaria coloniza el suelo o vegetales en descomposición y es capaz de producir conidias pequeñas e hidrofóbicas que se dispersan fácilmente en el aire y pueden sobrevivir a condiciones ambientales extremas ${ }^{1,2}$. Es un microorganismo comensal de la vía aérea; sin embargo, puede provocar un amplio espectro de patologías en el ser humano que van desde la hipersensibilidad de la vía aérea hasta la aspergilosis invasora por angio-invasión.

La aspergilosis invasora característicamente afecta a pacientes inmunocomprometidos, especialmente con neutropenia profunda y prolongada, pudiendo haber una invasión transbronquial, de los vasos sanguíneos pulmonares, hemorragias e infartos pulmonares con una mortalidad de hasta $86 \%$ incluso con tratamiento ${ }^{3,4}$. Otra forma clínica es la aspergilosis post-quirúrgica, que ocurre principalmente en pacientes inmunocompetentes, incluyendo aquellos pacientes que han recibido tratamiento corticoesteroidal en forma transitoria, y cuyo principal factor predisponente es la pérdida de la integridad de las barreras de la piel y mucosas durante la cirugía ${ }^{4-6}$. Es una complicación infrecuente pero grave de una cirugía, con una incidencia global de 2 en 10.000 procedimientos quirúrgicos ${ }^{6}$. La evolución clínica es generalmente indolente y se puede presentar meses después de la cirugía. Es causada por una fuente externa de conidias de Aspergillus spp. que contamina los tejidos del sitio operatorio durante o después del acto quirúrgico, y en la mayoría de los pacientes la fuente de las esporas parece estar originada en el ambiente del pabellón quirúrgico durante la cirugía. También se ha documentado la contaminación a través de los circuitos de diálisis, material quirúrgico contaminado (prótesis) o endógena por diseminación desde lesiones de otro sitio anatómico ${ }^{5-7}$.

Presentamos el caso de un paciente pediátrico que posterior a una cardiocirugía evoluciona con una mediastinitis por Aspergillus fumigatus.

\section{Caso clínico}

Escolar de 14 años, inmunocompetente, con antecedentes de una estenosis de la válvula pulmonar con prótesis valvular biológica y valvuloplastía de la tricúspide y portador de electrodos de marcapasos por un bloqueo atrio-ventricular.

Se había realizado recientemente un nuevo recambio de una válvula biológica y plastía de la válvula tricúspide. Cinco meses después consultó por cuatro días de aumento de volumen pre-esternal doloroso, sin fiebre. En ese momento, el hemograma tenía leucocitos de $14.800 / \mathrm{mm}^{3}$, y PCR de $40 \mathrm{mg} / \mathrm{L}$. Se realizó una ecografía de tejidos blandos que mostró un aumento del espesor y ecogenicidad del tejido celular subcutáneo pre-esternal, y varias colecciones en su interior, siendo la mayor de un volumen estimado de $10 \mathrm{ml}$. Se internó con el diagnóstico de mediastinitis y luego de obtener muestras para cultivo por punción pre-esternal se inició tratamiento antimicrobiano en forma empírica con vancomicina y meropenem i.v. En forma precoz se realizó aseo con drenaje quirúrgico, curetaje y extracción parcial de electrodo epicárdico. Durante la cirugía se evidenció compromiso del esternón,
Hospital Roberto del Río, Santiago, Chile (MA, FF, FC, DB).

Conflictos de interés: no existen. Financiamiento: No se recibió financiamiento externo.

Recibido: 15 de abril de 2015 Aceptado: 24 de noviembre de 2015

Correspondencia a: Mirta Acuña mirta.i.acuna@gmail.com 
confirmándose una mediastinitis con compromiso óseo e infección del marcapaso. Los hemocultivos, cultivos corrientes de la colección y del tejido no tuvieron desarrollo bacteriano. De la colección se realizaron RPC para Mycobacterium spp. y para Staphylococcus aureus resistente a meticilina (SARM) que fueron negativas. El paciente evolucionó en buen estado general, afebril. Se ajustó tratamiento antimicrobiano a vancomicina, cefotaxima y amikacina, completando 28 días en total. En forma ambulatoria completó dos semanas más con cefpodoxima y clindamicina por vía oral. Se realizó una TAC de tórax de control sin medio de contraste que mostró cambios post-quirúrgicos, una osteomielitis crónica esternal baja, sin colecciones residuales.

Un mes después consultó por dolor en la región subcostal derecha, sin fiebre. Una radiografía de esternón mostró un proceso inflamatorio inespecífico, y la ecografía de tejidos blandos demostró persistencia del compromiso del tejido celular subcutáneo esternal con colecciones en la profundidad del tejido subcutáneo y apéndice

Figura 1. TAC de tórax que muestra colecciones epicárdicas.
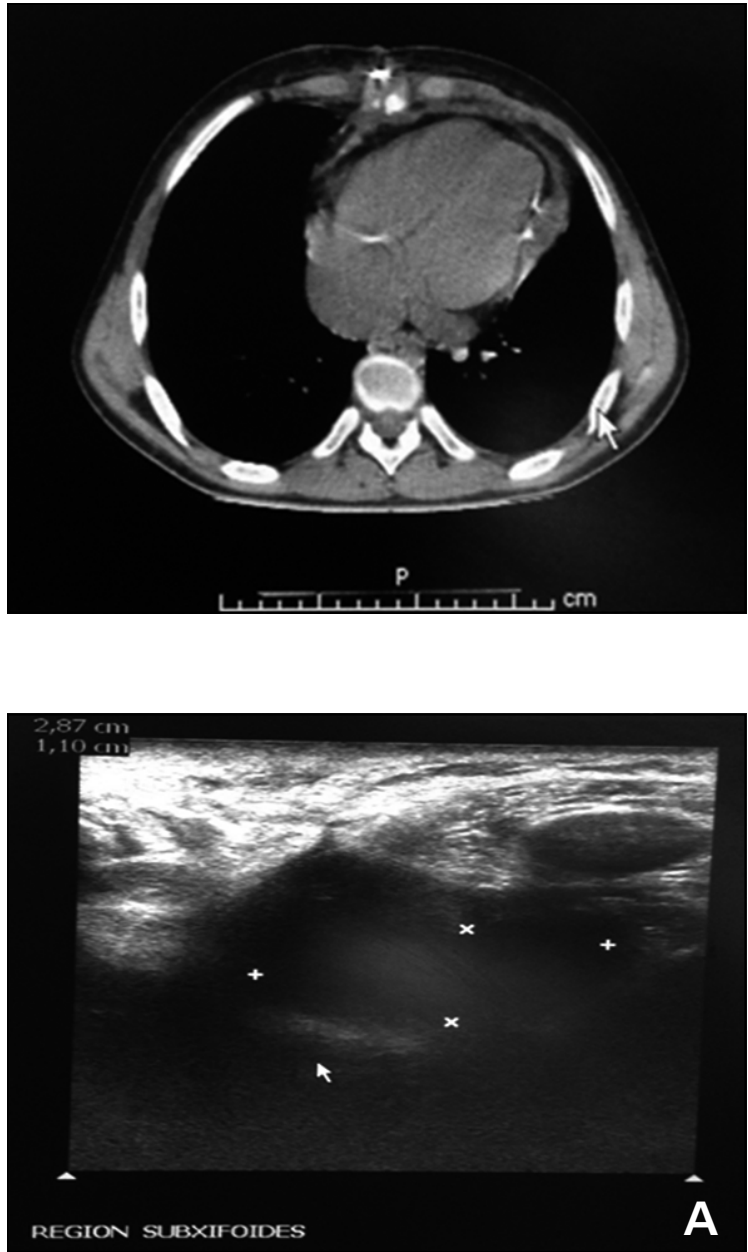

Figura 2. Ecografía de tórax que muestra lesión subxifoídea y preesternal, respectivamente. xifoides. El equipo tratante decidió observar la evolución. Posteriormente se controló una TAC de tórax, que mostró colecciones yuxtapericárdicas y una lesión lítica del extremo inferior del esternón (Figura 1). Se decidió reiniciar tratamiento antimicrobiano con clindamicina y ciprofloxacina por vía oral por cuatro semanas.

Aproximadamente un año después de la cirugía valvular presentó un nuevo aumento de volumen doloroso en la zona esternal. Una ecografía mostró una colección subcutánea de contenido heterogéneo, avascular y una colección retroesternal subxifoidea (Figura 2). Se obtuvo una muestra de la colección por punción que se inoculó en frascos de hemocultivos para aerobios, anaerobios y para micobacterias, tinción de Gram y baciloscopia. Fue sometido a una intervención quirúrgica para el retiro de los cables del marcapaso y aseo mediastínico, donde se obtuvieron muestras para cultivos de los cables y del tejido. Las muestras del tejido fueron sembradas en placas y tubos de agar Saboureaud gentamicina-cloranfenicol 2 (SGC2), Biomerieux, las cuales fueron incubadas a $35^{\circ} \mathrm{C}$ y a temperatura ambiente. Posterior a la cirugía, se inició tratamiento empírico con clindamicina y cefotaxima i.v. El vial de hemocultivo de la muestra de la colección fue positivo a las 54 h de incubación. En la tinción de Gram se observaron hifas, por lo que se inició anfotericina B deoxicolato. A las $72 \mathrm{~h}$ de siembra de las placas en agar Saboureaud se observó el desarrollo de colonias planas aterciopeladas y algodonosas de color verde en la superficie e incoloras en su reverso. En la observación microscópica realizada con azul de lactofenol, se encontraron hifas hialinas tabicadas, con presencia de cabezas aspergilares, con conidióforos incoloros lisos y regulares, vesículas en forma de mazo cubiertas en su tercio superior por fialides uniseriadas que daban origen a conidios lisos y globosos. Con estas características se confirmó A. fumigatus. Este resultado fue consistente con los aislados de las colecciones inoculadas en los frascos de hemocultivos (Figuras 3 y 4). Luego de tener

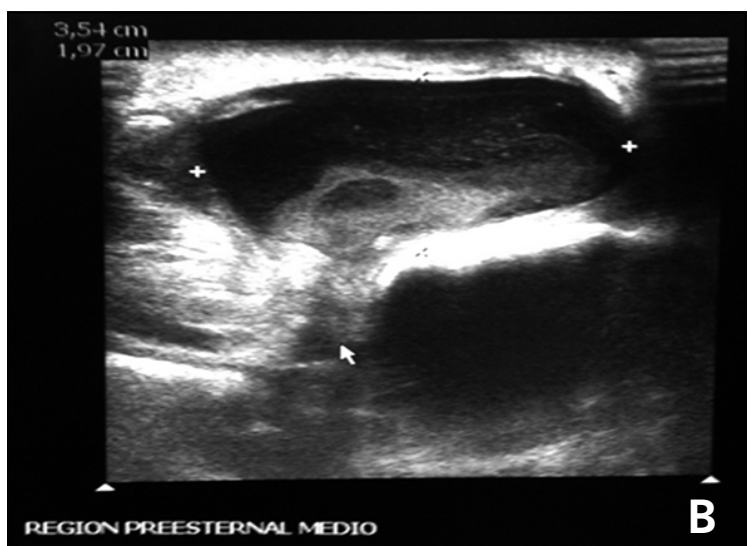


identificación de la especie se suspendieron definitivamente los antibacterianos y se ajustó el tratamiento antifúngico a voriconazol $200 \mathrm{mg}$ fraccionado cada 12 h. El paciente evolucionó en buenas condiciones con una TAC de tórax post-quirúrgica con pequeñas colecciones: yuxta-pericárdica y pre-esternal. Luego de una semana se decidió el alta con voriconazol con igual dosis. Durante el seguimiento ambulatorio el paciente se mantuvo clínicamente bien, con niveles plasmáticos de voriconazol adecuados. En el control imagenológico se observó una lenta regresión de las colecciones residuales. A los 10 meses de tratamiento con voriconazol, con una TAC de tórax con colecciones de menor tamaño en comparación con los estudios previos, se suspendió el antifúngico y se dio alta definitiva luego de 21 meses post-cirugía de recambio valvular, sin nuevas recidivas.

\section{Discusión}

El primer caso publicado de aspergilosis post-quirúrgica data de 1933, en una paciente sometida a una cirugía por un tumor abdominal que luego de 16 días presentó una úlcera en la herida operatoria con el hallazgo de Aspergillus niger en los apósitos quirúrgicos ${ }^{4}$. Nuestro paciente presentó una mediastinitis por $A$. fumigatus como una complicación de una cirugía cardíaca, coincidiendo con lo descrito en la literatura especializada. Las cirugías consideradas de alto riesgo de infección por Aspergillus spp. son las cirugías cardíacas, oftalmológicas y dentales ${ }^{6,7}$. Dentro de las infecciones post-cardiocirugía están la endocarditis, aortitis, osteomielitis esternal, la trombosis o la ruptura de la vena safena luego de una cirugía de by-pass. El período de incubación es variable, de menos de un mes hasta los 12 meses con una tasa de mortalidad hasta 92,7\%. El diagnóstico pre-mortem se logra sólo en 43,5\% de los $\operatorname{casos}^{4}$. Las infecciones del sitio quirúrgico (ISQ) por Aspergillus spp. se pueden presentar tan precozmente como a los días después de la cirugía, siendo casi la mitad pacientes sometidos a trasplante de órganos sólidos $^{4,6}$. La mediastinitis por Aspergillus spp. ha sido descrita en asociación con una infección profunda del sitio quirúrgico, en trasplante cardíaco, aortitis e infecciones del parche luego de la reparación de una tetralogía de Fallot $^{2,4,6,9-11}$. La ISQ por Aspergillus spp. tiende a ser destructiva, refractaria al tratamiento y las recaídas son frecuentes ${ }^{8}$. Es importante considerar la aspergilosis en el diagnóstico diferencial de las infecciones post-quirúrgicas de lenta progresión, destructivas y con cultivos corrientes negativos después de una cirugía cardíaca, tal como se presentó en nuestro paciente ${ }^{4,8,9}$.

El único factor de riesgo identificado para la ISQ por A. fumigatus en adultos es ser portador de enfermedad pulmonar crónica y estar colonizado previamente ${ }^{4}$.

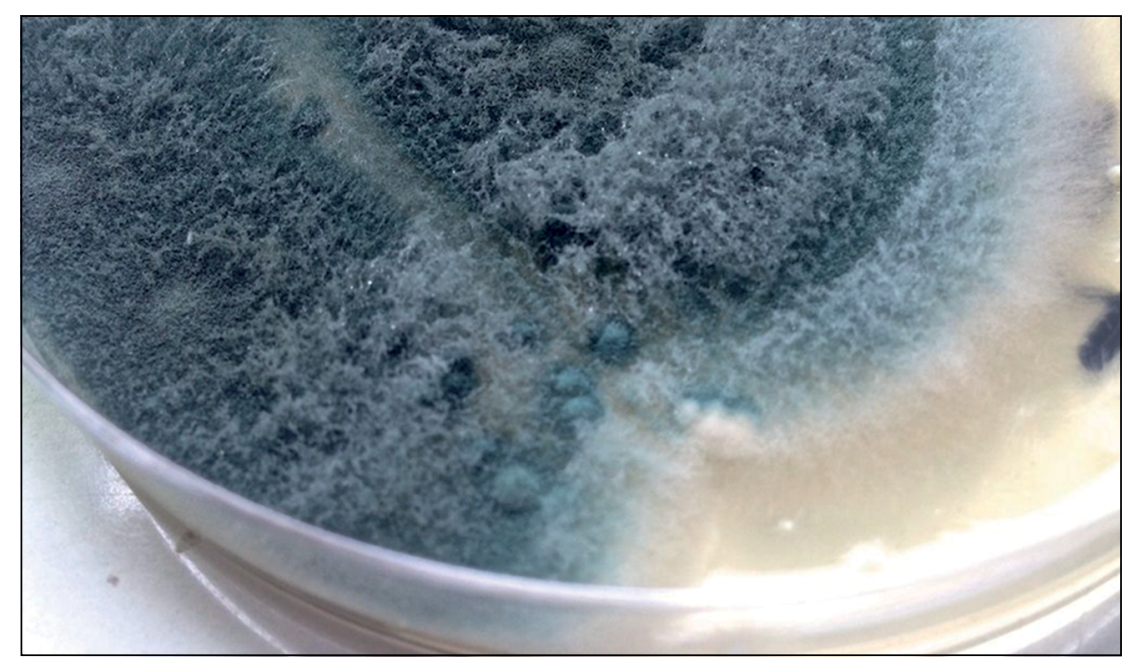

Figura 3. Placa de cultivo de Aspergillus fumigatus obtenida en el drenaje quirúrgico. Gentileza de T.M. Agustín Zamorano.

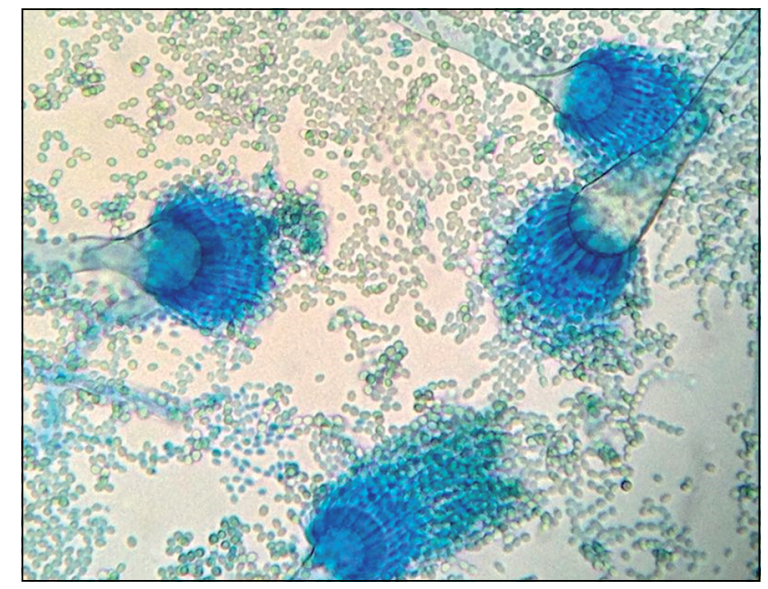

El tratamiento de las ISQ por Aspergillus spp. está basado en un enfrentamiento combinado médicoquirúrgico $^{8}$. El reconocimiento temprano de estas infecciones y el inicio precoz de tratamiento antifúngico asociado a la cirugía ha mejorado el pronóstico de estas infecciones ${ }^{5}$. Voriconazol es considerado el antifúngico de elección para el tratamiento de las infecciones por el género Aspergillus dado que prácticamente todas sus especies, incluido A. terreus, son susceptibles. Aunque no existen estudios prospectivos en este tipo de pacientes, las Guías de la Infectious Diseases Society of America (IDSA) recomiendan su uso para esta patología ${ }^{5,6,12}$. La duración del tratamiento antifúngico es aún desconocida. Se recomienda que una vez removido la mayor cantidad posible de tejido infectado, la terapia antifúngica debe ser administrada por un período no menor a tres meses a partir de la última evidencia de actividad de la infección ${ }^{6}$.
Figura 4. Microscopia de cultivo de Aspergillus fumigatus. Gentileza de T.M. Agustín Zamorano. 
Se han descrito brotes de infecciones por Aspergillus spp. asociados a construcciones dentro de los hospitales. Varias medidas de control de infecciones como la descontaminación ambiental, el uso de filtros de aire de alta eficiencia (HEPA) y medidas de mitigación de partículas de polvo en suspensión en las áreas en construcción han demostrado ser eficaces en la prevención de la adquisición nosocomial de aspergilosis ${ }^{7,9}$.

Los sistemas de flujo de aire laminar asociados a filtros de alta eficiencia (HEPA) son capaces de remover partículas en suspensión en un 99,97\%. Estos sistemas se utilizan en pabellones quirúrgicos y en aquellas unidades clínicas que requieran de un ambiente protegido por recibir pacientes de alto riesgo de infección por Aspergi$l l u s^{4,9}$. Pese a la seguridad del uso de filtros HEPA existen reportes de brotes de aspergilosis post-quirúrgica en unidades equipadas con filtros, por lo que la mantención de los sistemas de ventilación y el cambio de los filtros según el tiempo de saturación debe estar garantizada ${ }^{4}$.

Los métodos de tipificación molecular han sido utilizados para comparar las cepas de Aspergillus spp. de los pacientes con las muestras obtenidas del ambiente hospitalario. Cuando el aislado del paciente y la muestra ambiental tienen el mismo genotipo se puede establecer una fuente clonal única ambiental responsable del brote. Estos métodos son herramientas poderosas que tienen la capacidad de demostrar una clara asociación entre la fuente ambiental y los pacientes afectados y debe ser considerado en todo estudio de un brote ${ }^{4,7,9,11}$.

En nuestro paciente no se realizó muestreo y/o estudio ambiental dado que la infección se expresó clínicamente varios meses después de la intervención quirúrgica y se presentó como un caso único lo que hizo que el índice de sospecha de contaminación ambiental intrahospitalario fuera bajo.

\section{Conclusiones}

Se presenta el caso de una mediastinitis por A. fumigatus meses después de una cardiocirugía, de evolución tórpida y difícil diagnóstico que requirió de un alto índice de sospecha y múltiples cultivos para su confirmación. Una vez identificado el microorganismo responsable, con un tratamiento médico-quirúrgico adecuado, la respuesta terapéutica fue lenta pero favorable y sin recaídas.

En nuestro paciente la infección se presentó como un evento aislado por lo que no existió la sospecha ni la investigación concomitante para buscar una fuente ambiental; sin embargo, no puede descartarse que la contaminación inicial se produjo durante la primera cirugía.

Agradecimientos. A Dr. Luis Thompson, al equipo de cardiocirugía del Hospital Roberto del Río y a T.M. Agustín Zamorano.

\section{Referencias bibliográficas}

1.- Dagenais T R, Keller N P. Pathogenesis of Aspergillus fumigatus in invasive aspergillosis. Clin Microbiol Rev 2009; 22: 447-65.

2.- Ghotaslou R, Parvizi R, Safaei N, Yousefi S. A case of Aspergillus fumigatus mediastinitis after heart surgery in Madani Heart Center, Tabriz, Iran. Prog Cardiovasc Nurs 2008; 23: 133-5.

3.- Shakoor M T, Ayub S, Ayub Z, Mahmood F. Fulminant invasive aspergillosis of the mediastinum in an immunocompetent host: acase report. J Med Case Rep 2012; 6: 311

4.- Pasqualotto A C, Denning D W. Post-operative aspergillosis. Clin Microbiol Infect 2006; 12: 1060-76.

5.- Verghese S, Chellamma T, Cherian K M. Osteomyelitis of the rib caused by Aspergillus flavus following cardiac surgery. Mycoses 2009; 52: 91-3.
6.- Jensen J, Guinea J, Torres-Narbona M, Muñoz P, Peláez T, Bouza E. Post-surgical invasive aspergillosis: an uncommon and under-appreciated entity. J Infect 2010; 60: 162-7.

7.- Ruiz-Camps I, Aguado J M, Almirante B, Bouza E, Ferrer Barbera C, Len O, et al. Recomendaciones sobre la prevención de la infección fúngica invasora por hongos filamentosos de la Sociedad Española de Enfermedades Infecciosas y Microbiología Clínica (SEIMC). Enferm Infecc Microbiol Clin 2010; 28: 172. e1-172. e21.

8.- Vandecasteele S J, Boelaert J R, Verrelst P, Graulus E, Gordts B Z. Diagnosis and treatment of Aspergillus flavus sternal wound infections after cardiac surgery. Clin Infect Dis 2002; 35: 887-90.

9.- Kronman M P, Baden H P, Jeffries H E, Heath J, Cohen G A, Zerr D M. An investigation of
Aspergillus cardiac surgical site infections in 3 pediatric patients. Am J Infect Control 2007; 35: 332-7.

10.- Forestier E, Remy V, Lesens O, Martinot M, Hansman Y, Eisenmann B, et al. A case of Aspergillus mediastinitis after heart transplantation successfully treated with liposomal amphotericin B, caspofungin and voriconazole. Eur J Clin Microbiol Infect Dis 2005; 24: 347-9.

11.- Peláez T, Muñoz P, Guinea J, Valerio M, Giannella M, Klaassen C H, et al. Outbreak of invasive aspergillosis after major heart surgery caused by spores in the air of the intensive care unit. Clin Infect Dis 2012; 54: e24-31.

12.- Walsh T J, Anaissie E J, Denning D W, Herbrecht R, Kontoyiannis D P, Marr K A, et al. Treatment of aspergillosis: clinical practice guidelines of the Infectious Diseases Society of America. Clin Infect Dis 2008; 46: 327-60. 